\title{
HIGHLIGHTS
}

$\Rightarrow$ PROTEIN AGGREGATION

\section{Amyloid-directed phosphatase activation}

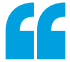 \\ amyloido- genesis of $\mathrm{Hzg}$ unleashes the phosphatase activity}

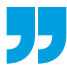

Prions are unique proteins that lack conformational stability and have a high propensity to aggregate. These aggregates may be dynamic, but can also take the form of highly insoluble, solid-like inclusions such as amyloids, which are generally stable. The formation of amyloids is strongly associated with pathology, in particular, degenerative diseases. Nil et al. now identify a new prionlike protein with phosphatase activity in Drosophila melanogaster and demonstrate that its assembly into amyloid-like aggregates is a physiological rather than a pathological process that is required for its enzymatic activity during fly development.

Recent analyses revealed that prion-like proteins are prevalent in eukaryotes, and there is now evidence that prions and prion-like proteins have various physiological functions. However, how aggregation affects the activity and biology of prions

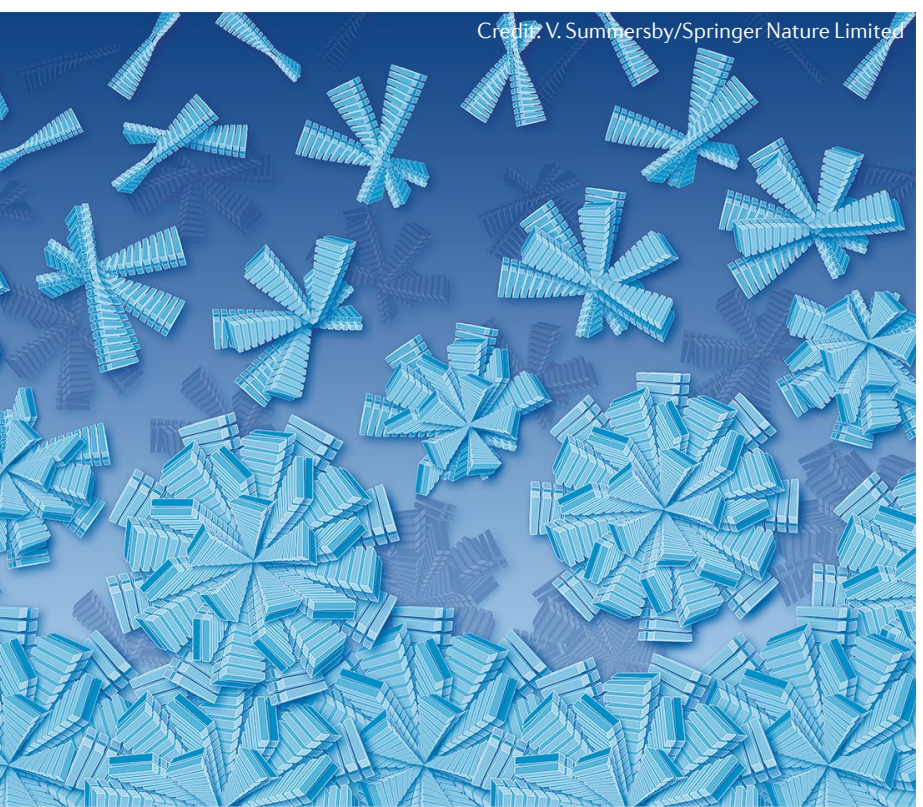

and prion-like proteins is elusive, particularly in metazoans. To fill this gap, the authors performed a proteome screen for new prion-like proteins in D. melanogaster and identified five proteins, including the previously uncharacterized protein Herzog (Hzg).

In fly embryos, Hzg was found to be membrane-associated and required for the expression of various patterning genes and embryonic development. In the course of embryonic development, the subcellular localization of $\mathrm{Hzg}$ was initially diffuse, but around gastrulation it was redistributed to distinct puncta at the plasma membrane. Biochemical analysis revealed that these post-gastrulation Hzg puncta were formed by highmolecular-weight aggregates in contrast to the low-molecularweight Hzg monomers present at early developmental stages - that demonstrated biochemical and biophysical properties of amyloids. Thus, Hzg forms amyloid-like aggregates in vivo and this amyloidogenesis is developmentally timed to coincide with gastrulation.

Hzg comprises a middle domain with homology to C-terminal domain RNA polymerase II phosphatase, flanked by two putative prion-like domains (PrDs). Ectopic expression of different Hzg constructs in the fly $\mathrm{S} 2$ cell line revealed that the N-terminal PrD is required for membrane localization and for conferring aggregation properties on Hzg.

Proteomic analyses indicated that Hzg aggregates interact with key developmental regulators, including components of TGF $\beta / \mathrm{BMP}$, EGF and FGF signalling pathways and with cell cycle-associated proteins.
One of the identified Hzg-aggregateinteracting cell cycle regulators, Dah, is a membrane-localized protein that undergoes dephosphorylation during gastrulation, suggesting that it may be regulated by Hzg aggregates. Indeed, ectopic expression of Hzg in S2 cells promoted Dah dephosphorylation. This apparent increase in phosphatase activity required $\mathrm{Hzg}$ targeting to the membrane and competence for aggregation. These data indicate that Hzg is an active phosphatase and that this activity depends on the formation of amyloid-like aggregates through the $\mathrm{N}$-terminal $\mathrm{PrD}$ at the cell membrane.

Next, in vitro analysis showed that high-molecular-weight Hzg aggregates purified from fly embryos were enzymatically active, whereas Hzg monomers were not. Furthermore, purified Hzg monomers allowed to self-assemble in vitro formed amyloid-like fibrils that accumulated over time. This aggregation was associated with a gradual increase in phosphatase activity, which correlated with the amount of amyloid-like Hzg fibrils. Finally, addition of an amyloid inhibitor to self-assembled Hzg aggregates significantly reduced phosphatase activity. Thus, amyloidogenesis of Hzg unleashes the phosphatase activity of the protein.

In summary, amyloidogenesis of Hzg is a developmentally programmed transition that is required for the activation of its phosphatase, which is required for patterning of the embryo. Moving forward, it will be interesting to study the exact mechanisms driving Hzg amyloidogenesis and how this process is regulated in development.

Paulina Strzyz

ORIGINAL ARTICLE Nil, Z. et al. Amyloid-like assembly activates a phosphatase in the developing Drosophila embryo. Cell https://doi.org/10.1016/ cell.2019.08.019 (2019)

RELATED ARTICLE ladanza, M. G. et al. A new era for understanding amyloid structures and disease. Nat. Rev. Mol. Cell Biol. 19, 755-773 (2018) 